\title{
On the global existence theorem \\ for a free boundary problem for equations of a viscous compressible heat conducting fluid
}

by EwA ZADRZYŃsKa and WoJCIECH M. ZAJA̧CZKOWski (Warszawa)

\begin{abstract}
We consider the motion of a viscous compressible heat conducting fluid in $\mathbb{R}^{3}$ bounded by a free surface which is under constant exterior pressure. Assuming that the initial velocity is sufficiently small, the initial density and the initial temperature are close to constants, the external force, the heat sources and the heat flow vanish, we prove the existence of global-in-time solutions which satisfy, at any moment of time, the properties prescribed at the initial moment.
\end{abstract}

1. Introduction. The main result of this paper is the global existence theorem for the following free boundary problem for a viscous compressible heat conducting fluid (see [4], Chs. 2 and 5):

$$
\begin{array}{ll}
\varrho\left[v_{t}+(v \cdot \nabla) v\right]+\nabla p-\mu \Delta v-\nu \nabla \operatorname{div} v=\varrho f & \text { in } \widetilde{\Omega}^{T}, \\
\varrho_{t}+\operatorname{div}(\varrho v)=0 & \text { in } \widetilde{\Omega}^{T}, \\
\varrho c_{v}\left(\theta_{t}+v \cdot \nabla \theta\right)+\theta p_{\theta} \operatorname{div} v-\kappa \Delta \theta & \\
\quad-\frac{\mu}{2} \sum_{i, j=1}^{3}\left(v_{i, x_{j}}+v_{j, x_{i}}\right)^{2}-(\nu-\mu)(\operatorname{div} v)^{2}=\varrho r & \text { in } \widetilde{\Omega}^{T}, \\
\mathbb{T} \cdot \bar{n}=-p_{0} \bar{n} & \text { on } \widetilde{S}^{T}, \\
v \cdot \bar{n}=-\frac{\varphi}{\mid \nabla \varphi} & \text { on } \widetilde{S}^{T}, \\
\frac{\partial \theta}{\partial n}=\theta_{1} & \text { on } \widetilde{S}^{T}, \\
\left.\varrho\right|_{t=0}=\varrho_{0},\left.\quad v\right|_{t=0}=v_{0},\left.\quad \theta\right|_{t=0}=\theta_{0} & \text { in } \Omega,
\end{array}
$$

where $\widetilde{\Omega}^{T}=\bigcup_{t \in(0, T)} \Omega_{t} \times\{t\}, \Omega_{t} \subset \mathbb{R}^{3}$ is a bounded domain depending

1991 Mathematics Subject Classification: 35A05, 35R35, 76N10.

Key words and phrases: viscous compressible heat conducting fluid, global existence, free boundary problem. 
on $t, \Omega_{0}=\Omega, \widetilde{S}^{T}=\bigcup_{t \in(0, T)} S_{t} \times\{t\}, S_{t}=\partial \Omega_{t}, \varphi(x, t)=0$ describes $S_{t}, \bar{n}$ is the unit outward vector normal to the boundary, i.e. $\bar{n}=\nabla \varphi /|\nabla \varphi|$. In (1.1), $v=v(x, t)$ is the velocity of fluid, $\varrho=\varrho(x, t)$ the density, $\theta=\theta(x, t)$ the temperature. Given functions are: $f=f(x, t)$, the external force field per unit mass; $r=r(x, t)$, the heat sources per unit mass; $\theta_{1}=\theta_{1}(x, t)$, the heat flow per unit surface; $p=p(\varrho, \theta)$, the pressure; $c_{v}=c_{v}(\varrho, \theta)$, the specific heat at constant volume. Moreover, $\mu$ and $\nu$ denote the viscosity coefficients, $\kappa$ the coefficient of the heat conductivity, and $p_{0}$ the external (constant) pressure. We assume that $\mu, \nu, \kappa$ are constants and thermodynamic considerations imply that $c_{v}>0, \kappa>0, \nu \geq \frac{1}{3} \mu>0$. Finally, $\mathbb{T}=\mathbb{T}(v, p)$ denotes the stress tensor of the form

$$
\begin{aligned}
\mathbb{T} & =\left\{T_{i j}\right\}=\left\{-p \delta_{i j}+\mu\left(v_{i, x_{j}}+v_{j, x_{i}}\right)+(\nu-\mu) \delta_{i j} \operatorname{div} v\right\} \\
& \equiv\left\{-p \delta_{i j}+D_{i j}(v)\right\},
\end{aligned}
$$

where $i, j=1,2,3$, and $\mathbb{D}=\mathbb{D}(v)=\left\{D_{i j}\right\}$ is the deformation tensor.

Let the domain $\Omega$ be given. Then by $(1.1)_{5}, \Omega_{t}=\left\{x \in \mathbb{R}^{3}: x=\right.$ $x(\xi, t), \xi \in \Omega\}$, where $x=x(\xi, t)$ is the solution of the Cauchy problem

$$
\frac{\partial x}{\partial t}=v(x, t),\left.\quad x\right|_{t=0}=\xi \in \Omega, \quad \xi=\left(\xi_{1}, \xi_{2}, \xi_{3}\right) .
$$

Therefore, we obtain the following relation between the Eulerian $x$ and the Lagrangian $\xi$ coordinates of the same fluid particle:

$$
x=\xi+\int_{0}^{t} u(\xi, s) d s \equiv X_{u}(\xi, t),
$$

where $u(\xi, t)=v\left(X_{u}(\xi, t), t\right)$. Moreover, the kinematic boundary condition $(1.1)_{5}$ implies that the boundary $S_{t}$ is a material surface. Thus, if $\xi \in S=S_{0}$ then $X_{u}(\xi, t) \in S_{t}$ and $S_{t}=\left\{x: x=X_{u}(\xi, t), \xi \in S\right\}$.

Equation of continuity $(1.1)_{2}$ and $(1.1)_{5}$ give the conservation of the total mass, i.e.

$$
\int_{\Omega_{t}} \varrho(x, t) d x=M
$$

In this paper we prove the existence of a global-in-time solution of problem (1.1) near a constant state.

To introduce the definition of the constant state consider the equation

$$
p\left(\varrho_{\mathrm{e}}, \theta_{\mathrm{e}}\right)=p_{0},
$$

where $\theta_{\mathrm{e}}=(1 /|\Omega|) \int_{\Omega} \theta_{0} d \xi$. We assume that equation (1.5) is solvable with respect to $\varrho_{\mathrm{e}}>0$. 
DeFInItion 1.1. Let $f=r=\theta_{1}=0$. Then by a constant (equilibrium) state we mean a solution $\left(v, \theta, \varrho, \Omega_{t}\right)$ of problem (1.1) such that $v=0$, $\varrho=\varrho_{\mathrm{e}}, \theta=\theta_{\mathrm{e}}, \Omega_{t}=\Omega_{\mathrm{e}}$ for $t \geq 0$, where $\varrho_{\mathrm{e}}$ is a solution of equation (1.5) and $\left|\Omega_{\mathrm{e}}\right|=M / \varrho_{\mathrm{e}}\left(\left|\Omega_{\mathrm{e}}\right|=\operatorname{vol} \Omega_{\mathrm{e}}\right)$.

The paper is divided into five sections. In Section 2 we introduce some notation and auxiliary results. In Section 3 we present the local existence theorem (see Theorem 3.1) proved in [16], while in Section 4 we recall the differential inequality (see Theorem 4.1) obtained in [19]. Finally, Section 5 is devoted to the global existence theorem (see Theorem 5.5).

The analogous problem to (1.1) for a viscous compressible barotropic fluid was considered by W. M. Zajączkowski in [20]. Hence, in order to prove Theorem 5.5 we apply a method similar to the proof of the global existence theorem in the barotropic case (see [20], Theorem 6.5). We prove Theorem 5.5 under the appropriate choice of $\varrho_{0}, v_{0}, \theta_{0}, \theta_{1}, p_{0}, \kappa$ and the form of the internal energy per unit mass $\varepsilon=\varepsilon(\varrho, \theta)$ (see conditions $(5.40)-(5.45)$ ) and under the assumption that $\varphi(0) \leq \varepsilon_{1}(\varphi(t)$ is given in $(4.5))$, where $\varepsilon_{1}$ is sufficiently small. In Theorem 5.5 we obtain a global solution of (1.1) such that $\left(v, \vartheta_{0}, \vartheta, \varrho_{\sigma}, \bar{\varrho}_{\Omega_{t}}\right) \in \mathfrak{M}(t)$ for $t \in \mathbb{R}_{+}^{1}$ (where $\vartheta_{0}, \vartheta, \varrho_{\sigma}, \bar{\varrho}_{\Omega_{t}}$ are defined in (4.2) and $\mathfrak{M}(t)$ is defined at the beginning of Section 5) and $S_{t} \in W_{2}^{4-1 / 2}$.

The papers [21]-[23] of W. M. Zajaczzkowski and the paper [14] of V. A. Solonnikov and A. Tani are devoted to the motion of a compressible barotropic viscous capillary fluid bounded by a free surface.

The motion of a viscous compressible heat conducting fluid in a fixed domain was considered by A. Matsumura and T. Nishida in [5]-[9] and by A. Valli and W. M. Zajạczkowski in [15], while the papers [11]-[13] of V. A. Solonnikov are concerned with free boundary problems for viscous incompressible fluids.

The papers [1], [2] of J. T. Beale are devoted to the global existence of solutions to free boundary problems, where the free boundary is unbounded and the gravitation is taken into account.

Problem (1.1) is considered also in the papers [16]-[19] of E. Zadrzyńska and W. M. Zajączkowski. In [16] the local existence of solutions to problem (1.1) is proved. In [18] conservation laws, and in [19] the differential inequality used in the proof of the global existence theorem are derived.

Finally, [17] is a survey of results concerning problem (1.1) and the free boundary problem with surface tension, analogous to problem (1.1).

2. Notation and auxiliary results. In Section 3 we use the anisotropic Sobolev-Slobodetskiǔ spaces $W_{2}^{l, l / 2}\left(Q_{T}\right), l \in \mathbb{R}_{+}^{1}$ (see [3]), of functions defined in $Q_{T}$, where $Q_{T}=\Omega^{T} \equiv \Omega \times(0, T)\left(\Omega \subset \mathbb{R}^{3}\right.$ is a domain, $T<\infty$ or $T=\infty)$ or $Q_{T}=S^{T} \equiv S \times(0, T), S=\partial \Omega$. 
We define $W_{2}^{l, l / 2}\left(\Omega^{T}\right)$ as the space of functions $u$ such that

$$
\begin{aligned}
\|u\|_{W_{2}^{l, l / 2}\left(\Omega^{T}\right)}= & {\left[\sum_{|\alpha|+2 i \leq[l]}\left\|D_{\xi}^{\alpha} \partial_{t}^{i} u\right\|_{L_{2}\left(\Omega^{T}\right)}^{2}\right.} \\
& +\sum_{|\alpha|+2 i=[l]}\left(\int_{0}^{T} \int_{\Omega} \int_{\Omega} \frac{\left|D_{\xi}^{\alpha} \partial_{t}^{i} u(\xi, t)-D_{\xi^{\prime}}^{\alpha} \partial_{t}^{i} u\left(\xi^{\prime}, t\right)\right|^{2}}{\left|\xi-\xi^{\prime}\right|^{3+2(l-[l])} d \xi d \xi^{\prime} d t}\right. \\
& +\int_{\Omega} \int_{0}^{T} \int_{0}^{T} \frac{\left|D_{\xi}^{\alpha} \partial_{t}^{i} u(\xi, t)-D_{\xi}^{\alpha} \partial_{t^{\prime}}^{i} u\left(\xi, t^{\prime}\right)\right|^{2}}{\left.\left.\left|t-t^{\prime}\right|^{1+2(l / 2-[l / 2])} d t d t^{\prime} d \xi\right)\right]^{1 / 2}<\infty,}
\end{aligned}
$$

where we use generalized (Sobolev) derivatives, $D_{\xi}^{\alpha}=\partial_{\xi_{1}}^{\alpha_{1}} \partial_{\xi_{2}}^{\alpha_{2}} \partial_{\xi_{3}}^{\alpha_{3}}$, $\partial_{\xi_{j}}^{\alpha_{j}}=$ $\partial^{\alpha_{j}} / \partial \xi_{j}^{\alpha_{j}}(j=1,2,3), \alpha=\left(\alpha_{1}, \alpha_{2}, \alpha_{3}\right)$ is a multiindex, $|\alpha|=\alpha_{1}+\alpha_{2}+\alpha_{3}$, $\partial_{t}^{i}=\partial^{i} / \partial t^{i}$ and $[l]$ is the integer part of $l$. In the case when $l$ is an integer the second terms in the above formulae must be omitted, while in the case of $l / 2$ being an integer the last terms in the above formulae must be omitted as well.

Similarly to $W_{2}^{l, l / 2}\left(\Omega^{T}\right)$, using local mappings and a partition of unity we introduce the normed space $W_{2}^{l, l / 2}\left(S^{T}\right)$ of functions defined on $S^{T}=$ $S \times(0, T)$, where $S=\partial \Omega$. We also use the ordinary Sobolev spaces $W_{2}^{l}(Q)$, where $l \in \mathbb{R}_{+}^{1}, Q=\Omega\left(\Omega \subset \mathbb{R}^{3}\right.$ is a bounded domain $)$ or $Q=S$. To simplify notation we write

$$
\begin{array}{ll}
\|u\|_{l, Q}=\|u\|_{W_{2}^{l, l / 2}(Q)} & \text { if } Q=\Omega^{T} \text { or } Q=S^{T}, \\
\|u\|_{l, Q}=\|u\|_{W_{2}^{l}(Q)} & \text { if } Q=\Omega \text { or } Q=S .
\end{array}
$$

Moreover, $\|u\|_{L_{p}(Q)}=|u|_{p, Q}, 1 \leq p \leq \infty$.

Now, we introduce the spaces $\Gamma_{k}^{l}(\Omega)$ and $\Gamma_{k}^{l, l / 2}(\Omega)$ of functions $u$ defined on $\Omega \times(0, T)(T<\infty$ or $T=\infty)$ such that

and

$$
|u|_{l, k, \Omega} \equiv\|u\|_{\Gamma_{k}^{l}(\Omega)}=\sum_{i \leq l-k}\left\|\partial_{t}^{i} u\right\|_{l-i, \Omega}<\infty
$$

$$
|u|_{l, k, \Omega} \equiv\|u\|_{\Gamma_{k}^{l, l / 2}(\Omega)}=\sum_{2 i \leq l-k}\left\|\partial_{t}^{i} u\right\|_{l-2 i, \Omega}<\infty,
$$

where $l \in \mathbb{R}_{+}^{1}, k \geq 0$.

Next, define the space $L_{p}\left(0, T ; \Gamma_{0}^{l, l / 2}(\Omega)\right.$ ) (where $1 \leq p \leq \infty$ ) with the norm $\|u\|_{L_{p}\left(0, T ; \Gamma_{0}^{l, l / 2}(\Omega)\right)}=|u|_{l, 0, p, \Omega^{T}}$.

Moreover, let $C^{2,1}(Q)$ (resp. $\left.C_{B}^{2,1}(Q)\right)\left(Q \subset \mathbb{R}^{3} \times[0, \infty)\right)$ denote the space of functions $u$ such that $D_{x}^{\alpha} \partial_{t}^{i} u \in C^{0}(Q)$ (resp. $\left.D_{x}^{\alpha} \partial_{t}^{i} u \in C_{B}^{0}(Q)\right)$ for $|\alpha|+2 i \leq 2\left(C_{B}^{0}(Q)\right.$ is the space of continuous bounded functions on $\left.Q\right)$. 
Finally, the following seminorm is used:

$$
|u|_{\kappa, Q^{T}}=\left(\int_{0}^{T} \frac{|u|_{2, Q}^{2}}{t^{2 \kappa}} d t\right)^{1 / 2}
$$

where $Q=\partial \Omega$.

Let $X$ be whichever of the function spaces mentioned above. We say that a vector-valued function $u=\left(u_{1}, \ldots, u_{\nu}\right)$ belongs to $X$ if $u_{i} \in X$ for any $1 \leq i \leq \nu$.

Moreover, we use the following lemmas.

LEMMA 2.1. The following imbedding holds:

$$
W_{r}^{l}(\Omega) \subset L_{p}^{\alpha}(\Omega) \quad\left(\Omega \subset \mathbb{R}^{3}\right),
$$

where $|\alpha|+3 / r-3 \leq l, l \in \mathbb{Z}, 1 \leq p, r \leq \infty ; L_{p}^{\alpha}(\Omega)$ is the space of functions $u$ such that $\left|D_{x}^{\alpha} u\right|_{p, \Omega}<\infty$, and $W_{r}^{l}(\Omega)$ is the Sobolev space.

Moreover, the following interpolation inequalities are true:

$$
\left|D_{x}^{\alpha} u\right|_{p, \Omega} \leq c \varepsilon^{1-\kappa}\left|D_{x}^{l} u\right|_{r, \Omega}+c \varepsilon^{-\kappa}|u|_{r, \Omega},
$$

where $\kappa=|\alpha| / l+3 /($ lr $)-3 /(l p)<1, \varepsilon$ is a parameter, and $c>0$ is a constant independent of $u$ and $\varepsilon$.

Lemma 2.1 follows from Theorem 10.2 of [3].

LEMMA 2.2 (see [10]). For a sufficiently regular u we have

$$
\left\|\partial_{t}^{i} u(t)\right\|_{2 l-1-2 i, \Omega} \leq c\left(\|u\|_{2 l, \Omega^{T}}+\left\|\partial_{t}^{i} u(0)\right\|_{2 l-1-2 i, \Omega}\right),
$$

where $0 \leq 2 i \leq 2 l-1, l \in \mathbb{N}$, and $c>0$ is a constant independent of $T$.

Now, consider problem (1.1). For (1.1) the energy conservation law is satisfied (see [4], Ch. 5).

Assume that the internal energy per unit mass $\varepsilon=\varepsilon(\varrho, \theta)$ has the form

$$
\varepsilon(\varrho, \theta)=a_{0} \varrho^{\alpha}+h(\varrho, \theta),
$$

where $a_{0}>0, \alpha>0, h(\varrho, \theta) \geq h_{*}>0, a_{0}, \alpha, h_{*}$ are constants and $h(\varrho, \theta)$ is a sufficiently regular function of its arguments. Moreover, we assume that $h(\varrho, \theta)$ has at $\left(\varrho_{\mathrm{e}}, \theta_{\mathrm{e}}\right)\left(\varrho_{\mathrm{e}}\right.$ and $\theta_{\mathrm{e}}$ are introduced in Definition 1.1) the only minimum point equal to $h_{*}$, i.e. $\min _{\varrho, \theta} h(\varrho, \theta)=h\left(\varrho_{\mathrm{e}}, \theta_{\mathrm{e}}\right)=h_{*}$.

In [19] it is shown that assumption (2.3) and the thermodynamical relation

$$
d \varepsilon=\theta d s+\frac{p}{\varrho^{2}} d \varrho
$$

(where $s$ is the density of entropy per unit mass) imply the following relations between $h, p$ and $c_{v}$ :

$$
\alpha a_{0} \varrho^{\alpha+1}+\varrho^{2} h_{\varrho}=p-\theta p_{\theta}
$$


and

$$
c_{v}=\frac{\partial \varepsilon}{\partial \theta}=h_{\theta} .
$$

In [18] (Corollary 1) the following result is proved.

LEMma 2.3. Let conditions (2.3)-(2.5) be satisfied. Let

$$
f=0, \quad \theta_{1} \geq 0 .
$$

Assume that

$$
\begin{gathered}
\int_{\Omega} \varrho_{0} \frac{v_{0}^{2}}{2} d \xi+\kappa \sup _{t} \int_{0}^{t} d t^{\prime} \int_{S_{t^{\prime}}} \theta_{1}\left(s, t^{\prime}\right) d s \\
\quad+\int_{\Omega} \varrho_{0} h\left(\varrho_{0}, \theta_{0}\right) d \xi-\inf _{t} \int_{\Omega_{t}} \varrho h(\varrho, \theta) d x \leq \delta_{0}, \\
\quad \int_{\Omega}\left|\varrho_{0}-\varrho_{\mathrm{e}}\right| d \xi \leq \delta_{0}, \\
\frac{(\beta-1)^{\beta-1}}{\beta^{\beta} p_{0}^{\beta-1}}\left(a_{0} \varrho_{\mathrm{e}}^{\beta}+p_{0}\right)^{\beta}-a_{0} \varrho_{\mathrm{e}}^{\beta} \leq \delta_{0} \\
a_{0}\left(\int_{\Omega} \varrho_{0}^{\beta} d \xi-\inf _{t} \int_{\Omega_{t}} \varrho^{\beta} d x\right) \leq \delta_{0},
\end{gathered}
$$

where $\delta_{0}>0$ is a sufficiently small constant and $\beta=\alpha+1$. Then

$$
\begin{array}{rl}
\frac{1}{2} \int_{\Omega_{t}} \varrho v^{2} & d x+a_{0}\left(\int_{\Omega_{t}} \varrho^{\beta} d x-\inf _{t} \int_{\Omega_{t}} \varrho^{\beta} d x\right) \\
& +\int_{\Omega_{t}} \varrho h(\varrho, \theta) d x-\inf _{t} \int_{\Omega_{t}} \varrho h(\varrho, \theta) d x+p_{0}\left(\left|\Omega_{t}\right|-\left|\Omega_{*}\right|\right) \leq c \widetilde{\delta},
\end{array}
$$

where $\left|\Omega_{*}\right|=\inf _{t}\left|\Omega_{t}\right|, c=$ const $>0$ is a constant and $\widetilde{\delta}=\widetilde{\delta}\left(\delta_{0}\right), \widetilde{\delta} \rightarrow 0$ as $\delta_{0} \rightarrow 0$.

Remark 2.4 (see [18], Theorem 2.7). Assumptions (2.3)-(2.9) imply that $\operatorname{var}_{t}\left|\Omega_{t}\right| \leq c \delta$, where $c>0$ is a constant, $\operatorname{var}_{t}\left|\Omega_{t}\right|=\sup _{t}\left|\Omega_{t}\right|-\inf _{t}\left|\Omega_{t}\right|$, $\delta^{2}=\widetilde{c} \delta_{0}$ and $\widetilde{c}$ is a constant.

Re mark 2.5. Since

$$
\int_{\Omega_{t}} \varrho h(\varrho, \theta) d x \geq h_{*} \int_{\Omega_{t}} \varrho d x=h_{*} M
$$

assumption (2.7) is satisfied if

$$
\begin{aligned}
\int_{\Omega} \varrho_{0} \frac{v_{0}^{2}}{2} d \xi+\kappa \sup _{t} & \int_{0}^{t} d t^{\prime} \int_{S_{t^{\prime}}} \\
& \theta_{1}\left(s, t^{\prime}\right) d s \\
& +\int_{\Omega} \varrho_{0}\left(h\left(\varrho_{0}, \theta_{0}\right)-h_{*}\right) d \xi \leq \delta_{0} .
\end{aligned}
$$


Re mark 2.6. By Remark 3 of [18] we have

$$
\begin{aligned}
& \frac{\left(d-\inf _{t} \int_{\Omega_{t}} \varrho h(\varrho, \theta) d x\right)(\beta-1)}{\beta p_{0}}-c \delta \\
& \leq\left|\Omega_{t}\right| \leq c \delta+\frac{\left(d-\inf _{t} \int_{\Omega_{t}} \varrho h(\varrho, \theta) d x\right)(\beta-1)}{\beta p_{0}}
\end{aligned}
$$

where $c>0$ is some constant, $\delta=\delta\left(\delta_{0}\right)$ with $\delta \rightarrow 0$ as $\delta_{0} \rightarrow 0$, and

$$
d=\int_{\Omega} \varrho_{0}\left(\frac{v_{0}^{2}}{2}+a_{0} \varrho_{0}^{2}+h\left(\varrho_{0}, \theta_{0}\right)\right) d \xi+p_{0}|\Omega|+\kappa \sup _{t} \int_{0}^{t} d t^{\prime} \int_{S_{t^{\prime}}} \theta_{1}\left(s, t^{\prime}\right) d s .
$$

Therefore, using (2.11) and the estimate

$$
\int_{\Omega_{t}} \varrho^{\beta} d x \geq\left|\Omega_{t}\right|^{1-\beta}\left(\int_{\Omega_{t}} \varrho d x\right)^{\beta}=\frac{M^{\beta}}{\left|\Omega_{t}\right|^{\beta-1}}
$$

we obtain

$$
\inf _{t} \int_{\Omega_{t}} \varrho^{\beta} d x \geq \frac{M^{\beta}\left(\beta p_{0}\right)^{\beta-1}}{\left[c \delta \beta \varrho_{0}+\left(d-h_{*} M\right)(\beta-1)\right]^{\beta-1}} .
$$

Hence, assumption (2.10) is satisfied if

$$
a_{0}\left\{\int_{\Omega} \varrho_{0}^{\beta} d x-\frac{M^{\beta}\left(\beta p_{0}\right)^{\beta-1}}{\left[c \delta \beta \varrho_{0}+\left(d-h_{*} M\right)(\beta-1)\right]^{\beta-1}}\right\} \leq \delta_{0} .
$$

We see that the left-hand side of (2.13) tends to 0 as $\beta \rightarrow 1$, so for $\beta$ sufficiently close to 1 , it is as small as we wish.

3. Local existence. To prove the local existence for (1.1) we rewrite it in the Lagrangian coordinates introduced by (1.2) and (1.3):

$$
\begin{array}{ll}
\eta u_{t}-\mu \nabla_{u}^{2} u-\nu \nabla_{u} \nabla_{u} \cdot u+\nabla_{u} p(\eta, \Gamma)=\eta g & \text { in } \Omega^{T} \equiv \Omega \times(0, T), \\
\eta_{t}+\eta \nabla_{u} \cdot u=0 & \text { in } \Omega^{T}, \\
\eta c_{v}(\eta, \Gamma) \Gamma_{t}-\kappa \nabla_{u}^{2} \Gamma=-\Gamma p_{\Gamma}(\eta, \Gamma) \nabla_{u} \cdot u & \\
\quad+\frac{\mu}{2} \sum_{i, j=1}^{3}\left(\xi_{x_{i}} \nabla_{\xi} u_{j}+\xi_{x_{j}} \nabla_{\xi} u_{i}\right)^{2} & \\
\quad+(\nu-\mu)\left(\nabla_{u} \cdot u\right)^{2}+\eta k & \text { in } \Omega^{T}, \\
\mathbb{T}_{u}(u, p) \cdot \bar{n}=-p_{0} \bar{n} & \text { on } S^{T}, \\
\bar{n} \cdot \nabla_{u} \Gamma=\Gamma_{1} & \text { on } S^{T}, \\
\left.u\right|_{t=0}=v_{0} & \text { in } \Omega, \\
\left.\eta\right|_{t=0}=\varrho_{0} & \text { in } \Omega, \\
\left.\Gamma\right|_{t=0}=\theta_{0} & \text { in } \Omega,
\end{array}
$$


where $u(\xi, t)=v\left(X_{u}(\xi, t), t\right), \Gamma(\xi, t)=\theta\left(X_{u}(\xi, t), t\right), \eta(\xi, t)=\varrho\left(X_{u}(\xi, t), t\right)$, $g(\xi, t)=f\left(X_{u}(\xi, t), t\right), k(\xi, t)=r\left(X_{u}(\xi, t), t\right), \nabla_{u}=\xi_{x} \nabla_{\xi} \equiv\left\{\xi_{i x} \partial_{\xi_{i}}\right\}$, $\mathbb{T}_{u}(u, p)=-p I+\mathbb{D}_{u}(u), \mathbb{D}_{u}(u)=\left\{\mu\left(\xi_{k x_{i}} \partial_{\xi_{k}} u_{j}+\xi_{k x_{j}} \partial_{\xi_{k}} u_{i}\right)+(\nu-\mu) \delta_{i j} \nabla_{u} u\right\}$ (here the summation convention over the repeated indices is assumed), and $\Gamma_{1}(\xi, t)=\theta_{1}\left(X_{u}(\xi, t), t\right)$.

Let $A=\left\{a_{i j}\right\}$ be the Jacobi matrix of the transformation $x=X_{u}(\xi, t)$, where $a_{i j}=\delta_{i j}+\int_{0}^{t} \partial_{\xi_{j}} u_{i}\left(\xi, t^{\prime}\right) d t^{\prime}$. Assuming that $\left|\nabla_{\xi} u\right|_{\infty, \Omega^{T}} \leq M$ we obtain

$$
0<c_{1}(1-M t)^{3} \leq \operatorname{det}\left\{x_{\xi}\right\} \leq c_{2}(1+M t)^{3}, \quad t \leq T,
$$

where $c_{1}, c_{2}>0$ are constants and $T>0$ is sufficiently small. Moreover, $\operatorname{det} A=\exp \left(\int_{0}^{t} \nabla_{u} u d t^{\prime}\right)=\varrho_{0} / \eta$.

Let $S_{t}$ be determined (at least locally) by the equation $\varphi(x, t)=0$. Then $S$ is described by $\left.\varphi(x(\xi, t), t)\right|_{t=0}=\widetilde{\varphi}(\xi)=0$. Thus, we have

$$
\bar{n}(x(\xi, t), t)=-\left.\frac{\nabla_{x} \varphi(x, t)}{\left|\nabla_{x} \varphi(x, t)\right|}\right|_{x=x(\xi, t)} \quad \text { and } \quad \bar{n}_{0}(\xi)=-\frac{\nabla_{\xi} \widetilde{\varphi}(\xi)}{\left|\nabla_{\xi} \widetilde{\varphi}(\xi)\right|} .
$$

Now, we are able to formulate the local existence theorem.

Theorem 3.1 (see [16], Theorem 3.7). Let $S \in W_{2}^{4-1 / 2}, f \in C^{2,1}\left(\mathbb{R}^{3} \times\right.$ $[0, T]), r \in C^{2,1}\left(\mathbb{R}^{3} \times[0, T]\right), \theta_{1} \in C^{2,1}\left(\mathbb{R}^{3} \times[0, T]\right), v_{0} \in W_{2}^{3}(\Omega), \theta_{0} \in$ $W_{2}^{3}(\Omega), 1 / \theta_{0} \in L_{\infty}(\Omega), \theta_{0}>0, \varrho_{0} \in W_{2}^{3}(\Omega), 1 / \varrho_{0} \in L_{\infty}(\Omega), \varrho_{0}>0$, $c_{v} \in C^{2}\left(\mathbb{R}_{+}^{2}\right), c_{v}>0, p \in C^{3}\left(\mathbb{R}_{+}^{2}\right)$. Moreover, assume that the following compatibility conditions are satisfied:

$$
D_{\xi}^{\alpha}\left(\mathbb{D}_{\xi}\left(v_{0}\right) \cdot \bar{n}_{0}-p\left(\varrho_{0}, \theta_{0}\right) \bar{n}_{0}\right)=-D_{\xi}^{\alpha}\left(p_{0} \bar{n}_{0}\right), \quad|\alpha| \leq 1, \quad \text { on } S
$$

and

$$
D_{\xi}^{\alpha}\left(\bar{n}_{0} \cdot \nabla_{\xi} \theta_{0}\right)=D_{\xi}^{\alpha}\left(\theta_{1}(\xi, 0)\right), \quad|\alpha| \leq 1, \quad \text { on } S .
$$

Let $T^{*}>0$ be so small that $0<c_{1}\left(1-C K_{0} T^{*}\right)^{3} \leq \operatorname{det}\left\{x_{\xi}\right\} \leq c_{2}\left(1+C K_{0} T^{*}\right)^{3}$ (where $x(\xi, t)=\xi+\int_{0}^{t} u_{0}\left(\xi, t^{\prime}\right) d t^{\prime}$ for $t \leq T^{*}, u_{0}$ is given by (3.74) of [16], $K_{0} \leq c\left(\left\|\varrho_{0}\right\|_{3, \Omega}+\left|\varrho_{0}\right|_{\infty, \Omega}+\left|1 / \varrho_{0}\right|_{\infty, \Omega}+\left\|v_{0}\right\|_{3, \Omega}+\left\|\theta_{0}\right\|_{3, \Omega}+\left\|u_{t}(0)\right\|_{1, \Omega}+\right.$ $\left.\left\|\Gamma_{t}(0)\right\|_{1, \Omega}\right), c>0$ is a constant, $C=C\left(K_{0}\right)$ is a nondecreasing continuous function of $K_{0}$ satisfying (3.94) of [16]). Then there exists $T^{* *}$ with $0<$ $T^{* *} \leq T^{*}$ such that for $T \leq T^{* *}$ there exists a unique solution $(u, \Gamma, \eta) \in$ $W_{2}^{4,2}\left(\Omega^{T}\right) \times W_{2}^{4,2}\left(\Omega^{T}\right) \times C^{0}\left(0, T ; \Gamma_{0}^{3,3 / 2}(\Omega)\right)$ of problem (3.1). Moreover, $\eta_{t} \in C^{0}\left(0, T ; W_{2}^{2}(\Omega)\right) \cap L_{2}\left(0, T ; W_{2}^{3}(\Omega)\right), \eta_{t t} \in L_{2}\left(0, T ; W_{2}^{1}(\Omega)\right)$ and $\|u\|_{4, \Omega^{T}}+\|\Gamma\|_{4, \Omega^{T}} \leq C K_{0}$, $\sup _{t}\|\eta\|_{3, \Omega}+\sup _{t}\left\|\eta_{t}\right\|_{2, \Omega}+\left\|\eta_{t}\right\|_{L_{2}\left(0, T ; W_{2}^{3}(\Omega)\right)}$

$$
+\left\|\eta_{t t}\right\|_{L_{2}\left(0, T ; W_{2}^{1}(\Omega)\right)} \leq \Phi_{1}\left(T, T^{a} K_{0}\right)\left\|\varrho_{0}\right\|_{3, \Omega},
$$

$|1 / \eta|_{\infty, \Omega^{T}}+|\eta|_{\infty, \Omega^{T}}$

$$
\leq \Phi_{2}\left(T^{1 / 2} K_{0}\right)\left|1 / \varrho_{0}\right|_{\infty, \Omega}+\Phi_{3}\left(T^{1 / 2} K_{0}\right)\left|\varrho_{0}\right|_{\infty, \Omega},
$$

where $\Phi_{1}, \Phi_{2}$ and $\Phi_{3}$ are increasing continuous functions, and $a>0$. 
In order to consider the global existence we need

R e m ark 3.2. Assume that $g=0$ and define

$$
p_{\sigma}=p-p_{0}, \quad \gamma_{0}=\Gamma-\theta_{\mathrm{e}}, \quad \eta_{\sigma}=\eta-\varrho_{\mathrm{e}}
$$

(where $\theta_{\mathrm{e}}$ and $\varrho_{\mathrm{e}}$ are introduced in Definition 1.1). Then problem (3.1) can be written in the form

$$
\begin{aligned}
& \eta u_{t}-\mu \nabla_{u}^{2} u-\nu \nabla_{u} \nabla_{u} \cdot u+\nabla_{u} p_{\sigma}=0 \\
& \eta_{\sigma t}+\eta \nabla_{u} \cdot u=0 \\
& \eta c_{v}(\eta, \Gamma) \gamma_{0 t}-\kappa \nabla_{u}^{2} \gamma_{0}+\Gamma p_{\Gamma}(\eta, \Gamma) \nabla_{u} \cdot u \\
& \quad-\frac{\mu}{2} \sum_{i, j=1}^{3}\left(\xi_{x_{i}} \nabla_{\xi} u_{j}+\xi_{x_{j}} \nabla_{\xi} u_{i}\right)^{2}-(\nu-\mu)\left(\nabla_{u} \cdot u\right)^{2}=\eta k, \\
& \mathbb{T}_{u}\left(u, p_{\sigma}\right) \cdot \bar{n}=0, \\
& \bar{n} \cdot \nabla_{u} \gamma_{0}=\Gamma_{1}, \\
& \left.u\right|_{t=0}=v_{0},\left.\quad \eta_{\sigma}\right|_{t=0}=\varrho_{\sigma 0},\left.\quad \gamma_{0}\right|_{t=0}=\vartheta_{00},
\end{aligned}
$$

where $\varrho_{\sigma 0}=\varrho_{0}-\varrho_{\mathrm{e}}$ and $\vartheta_{00}=\theta_{0}-\theta_{\mathrm{e}}$.

Let the assumptions of Theorem 3.1 be satisfied and let $(u, \Gamma, \eta)$ be the corresponding local solution of problem (3.1). Then by Theorems 3.5, 3.6 and Lemma 3.3 of [16] for a solution $\left(u, \gamma_{0}, \eta_{\sigma}\right)$ of $(3.5)$ such that

$$
T^{a}\left(\|u\|_{4, \Omega^{T}}+\left\|v_{0}\right\|_{3, \Omega}+\left\|\vartheta_{00}\right\|_{3, \Omega}+\left\|\varrho_{\sigma 0}\right\|_{3, \Omega}\right) \varphi_{1}\left(T, K_{0}\right) \leq \delta
$$

(where $a>0$ is a constant, $\varphi_{1}$ is an increasing continuous function of its arguments, $\delta>0$ is sufficiently small) the following estimate holds:

$$
\begin{aligned}
\|u\|_{4, \Omega^{T}} & +\left\|\eta_{\sigma}\right\|_{3, \Omega^{T}}+\left|\eta_{\sigma}\right|_{3,0, \infty, \Omega^{T}}+\left\|\gamma_{0}\right\|_{4, \Omega^{T}} \\
\leq & \varphi_{2}\left(T, K_{0}\right)\left(\left\|v_{0}\right\|_{3, \Omega}+\left\|\varrho_{\sigma 0}\right\|_{3, \Omega}+\left\|\vartheta_{00}\right\|_{3, \Omega}\right. \\
& \left.\quad+\|k\|_{2, \Omega^{T}}+\|k(0)\|_{1, \Omega}+\left\|\Gamma_{1}\right\|_{3-1 / 2, S^{T}}+\mid D_{\xi, t}^{2} \Gamma_{1} \mathbf{l}_{1 / 4, S^{T}}\right)
\end{aligned}
$$

where $\varphi_{2}$ is an increasing continuous function of its arguments.

4. Differential inequality. In order to prove the global existence of solutions we need the differential inequality derived in [19] (Theorem 3.13). Assume that the existence of a sufficiently smooth local solution of problem (1.1) has been proved and consider the motion near the constant state (see Definition 1.1) $v_{\mathrm{e}}=0, p_{\mathrm{e}}=p_{0}, \theta_{\mathrm{e}}=(1 /|\Omega|) \int_{\Omega} \theta_{0} d \xi$ and $\varrho_{\mathrm{e}}$, where $\varrho_{\mathrm{e}}$ is a solution of the equation

$$
p\left(\varrho_{\mathrm{e}}, \theta_{\mathrm{e}}\right)=p_{0} .
$$


Let

$$
\begin{aligned}
& p_{\sigma}=p-p_{0}, \quad \varrho_{\sigma}=\varrho-\varrho_{\mathrm{e}}, \quad \vartheta_{0}=\theta-\theta_{\mathrm{e}}, \\
& \vartheta=\theta-\theta_{\Omega_{t}}, \quad \bar{\varrho}_{\Omega_{t}}=\varrho-\varrho_{\Omega_{t}},
\end{aligned}
$$

where $\theta_{\Omega_{t}}=\left(1 /\left|\Omega_{t}\right|\right) \int_{\Omega_{t}} \theta d x$, and $\varrho_{\Omega_{t}}=\varrho_{\Omega_{t}}(t)$ is a solution of the problem

$$
p\left(\varrho_{\Omega_{t}}, \theta_{\Omega_{t}}\right)=p_{0},\left.\quad \varrho_{\Omega_{t}}\right|_{t=0}=\varrho_{\mathrm{e}}
$$

Then problem (1.1) takes the form

$$
\begin{array}{ll}
\varrho\left[v_{t}+(v \cdot \nabla) v\right]-\operatorname{div} \mathbb{T}\left(v, p_{\sigma}\right)=\varrho f & \text { in } \Omega_{t}, t \in[0, T], \\
\varrho \varrho_{t}+\operatorname{div}(\varrho v)=0 & \text { in } \Omega_{t}, t \in[0, T], \\
\varrho c_{v}(\varrho, \theta)\left(\vartheta_{0 t}+v \cdot \nabla \vartheta_{0}\right)+\theta p_{\theta}(\varrho, \theta) \operatorname{div} v & \\
\quad-\kappa \Delta \vartheta_{0}-\frac{\mu}{2} \sum_{i, j}\left(\partial_{x_{i}} v_{j}+\partial_{x_{j}} v_{i}\right)^{2} & \\
\quad-(\nu-\mu)(\operatorname{div} v)^{2}=\varrho r & \operatorname{in} \Omega_{t}, t \in[0, T], \\
\mathbb{T}\left(v, p_{\sigma}\right) \cdot \bar{n}=0 & \text { on } S_{t}, t \in[0, T], \\
\partial \vartheta_{0} / \partial n=\theta_{1} & \text { on } S_{t}, t \in[0, T],
\end{array}
$$

where $\mathbb{T}\left(v, p_{\sigma}\right)=\left\{\mu\left(\partial_{x_{i}} v_{j}+\partial_{x_{j}} v_{i}\right)+(\nu-\mu) \delta_{i j} \operatorname{div} v-p_{\sigma} \delta_{i j}\right\}$ and $T$ is the time of the local existence.

Define

$$
\begin{aligned}
\bar{\varphi}(t)= & \int_{\Omega_{t}} \varrho \sum_{1 \leq|\alpha|+i \leq 3}\left|D_{x}^{\alpha} \partial_{t}^{i} v\right|^{2} d x \\
& +\int_{\Omega_{t}}\left(\frac{p_{1}}{\varrho} \varrho_{\sigma}^{2}+\bar{\varrho}_{\Omega_{t}}^{2}+\frac{p_{2} \varrho c_{v}}{p_{\theta} \theta} \vartheta_{0}^{2}\right) d x \\
& +\int_{\Omega_{t}} \frac{p_{\sigma \varrho}}{\varrho} \sum_{1 \leq|\alpha|+i \leq 3}\left|D_{x}^{\alpha} \partial_{t}^{i} \varrho_{\sigma}\right|^{2} d x \\
& +\int_{\Omega_{t}} \frac{\varrho c_{v}}{\theta} \sum_{1 \leq|\alpha|+i \leq 3}\left|D_{x}^{\alpha} \partial_{t}^{i} \vartheta_{0}\right|^{2} d x, \\
\varphi(t)= & |v|_{3,0, \Omega_{t}}^{2}+\left|\vartheta_{0}\right|_{3,0, \Omega_{t}}^{2}+\left|\varrho_{\sigma}\right|_{3,0, \Omega_{t}}^{2}+\|\left.\bar{\varrho}_{\Omega_{t}}\right|_{0, \Omega_{t}} ^{2}, \\
\Phi(t)= & |v|_{4,1, \Omega_{t}}^{2}+\left|\vartheta_{0}\right|_{4,1, \Omega_{t}}^{2}-\left\|\vartheta_{0}\right\|_{0, \Omega_{t}}^{2}+\|\vartheta\|_{0, \Omega_{t}}^{2} \\
& +\left|\varrho_{\sigma}\right|_{3,0, \Omega_{t}}^{2}-\left\|\varrho_{\sigma}\right\|_{0, \Omega_{t}}^{2}+\left\|\bar{\varrho}_{\Omega_{t}}\right\|_{0, \Omega_{t}}^{2}, \\
F(t)= & \left\|f_{t t t}\right\|_{0, \Omega_{t}}^{2}+|f|_{2,0, \Omega_{t}}^{2}+\left\|r_{t t t}\right\|_{0, \Omega_{t}}^{2}+|r|_{2,0, \Omega_{t}}^{2} \\
& +\|r\|_{0, \Omega_{t}}+\left|\theta_{1}\right|_{4,1, \Omega_{t}}^{2}+\left\|\theta_{1}\right\|_{1, \Omega_{t}}, \\
\psi(t)= & \|v\|_{0, \Omega_{t}}^{2}+\left\|p_{\sigma}\right\|_{0, \Omega_{t}}^{2} .
\end{aligned}
$$

The following theorem is proved in [19] (see Theorem 3.13). 
THEOREM 4.1. Let $\nu>\frac{1}{3} \mu$. Then for a sufficiently smooth solution $(v$, $\left.\vartheta_{0}, \varrho\right)$ of (4.4) we have

$$
\begin{aligned}
\frac{d \bar{\varphi}}{d t}+c_{0} \Phi \leq & c_{1} P(\varphi)\left(\varphi+\int_{0}^{t}\|v\|_{4, \Omega_{t^{\prime}}}^{2} d t^{\prime}\right)\left(1+\varphi^{3}\right)(\varphi+\Phi) \\
& +c_{2} F+c_{3} \psi
\end{aligned}
$$

where $P$ is an increasing continuous function; $0<c_{0}<1$ is a constant depending on $\varrho^{*}, \varrho_{*}, \theta^{*}, \theta_{*}, \mu, \nu, \kappa$; and $c_{i}(i=1,2,3)$ are positive constants depending on $\varrho_{*}, \varrho^{*}, \theta_{*}, \theta^{*}, \int_{0}^{t}\|v\|_{3, \Omega_{t^{\prime}}} d t^{\prime},\|S\|_{4-1 / 2}, T$ and the constants from the imbedding Lemma 2.1 and the Korn inequalities from [20] (Section 5).

Remark 4.2. Theorem 3.13 of [20] was proved under the assumption that $\nu \geq \mu$. This assumption implies that

$$
\frac{\mu}{2} E_{\Omega_{t}}(v)+(\nu-\mu)\|\operatorname{div} v\|_{0, \Omega_{t}}^{2} \geq 0,
$$

where $E_{\Omega_{t}}(v)=\int_{\Omega_{t}} \sum_{i, j=1}^{3}\left(v_{i, x_{j}}+v_{j, x_{i}}\right)^{2}$.

It turns out that the condition $\nu \geq \mu$ is too restrictive and we can now show that (4.7) is satisfied for $\nu>\frac{1}{3} \mu$, which is assumed in Theorem 4.1. In fact, we have

$$
\begin{aligned}
\frac{\mu}{2} E_{\Omega_{t}}(v) & +(\nu-\mu)\|\operatorname{div} v\|_{0, \Omega_{t}}^{2} \\
= & \frac{\mu}{2} \int_{\Omega_{t}}\left(v_{i, x_{j}}+v_{j, x_{i}}\right)^{2} d x+(\nu-\mu) \int_{\Omega_{t}}(\operatorname{div} v)^{2} d x \\
= & \frac{\mu}{2} \sum_{i \neq j} \int_{\Omega_{t}}\left(v_{i, x_{j}}+v_{j, x_{i}}\right)^{2} d x+\frac{\mu}{2} \sum_{i=j} \int_{\Omega_{t}}\left(v_{i, x_{j}}+v_{j, x_{i}}\right)^{2} d x \\
& +(\nu-\mu) \int_{\Omega_{t}}(\operatorname{div} v)^{2} d x \\
= & \frac{\mu}{2} \sum_{i \neq j} \int_{\Omega_{t}}\left(v_{i, x_{j}}+v_{j, x_{i}}\right)^{2} d x \\
& +\frac{\mu}{2} \varepsilon_{1} \sum_{i=j} \int_{\Omega_{t}}\left(v_{i, x_{j}}+v_{j, x_{i}}\right)^{2} d x \\
& +\frac{\mu}{2}\left(1-\varepsilon_{1}\right) \cdot 4 \sum_{i} \int_{\Omega_{t}}\left(v_{i, x_{j}}\right)^{2} d x \\
& +(\nu-\mu) \int_{\Omega_{t}}(\operatorname{div} v)^{2} d x \equiv I
\end{aligned}
$$

where $\varepsilon_{1} \in(0,1)$. 
Since $\left(\xi_{1}+\xi_{2}+\xi_{3}\right)^{2} \leq 3\left(\xi_{1}^{2}+\xi_{2}^{2}+\xi_{3}^{2}\right)$ the last two terms in $I$ are estimated from below by

$$
\left[\nu-\left(1+2 \varepsilon_{1}\right) \frac{\mu}{2}\right] \int_{\Omega_{t}}(\operatorname{div} v)^{2} d x .
$$

Assuming that $\nu=\left(1+2 \varepsilon_{1}\right) \mu / 3$ we obtain $\varepsilon_{1}=\frac{3}{2 \mu}(\nu-\mu / 3)$, so

$$
\begin{aligned}
I & \geq \frac{\mu}{2} \varepsilon_{1} \int_{\Omega_{t}}\left(v_{i, x_{j}}+v_{j, x_{i}}\right)^{2} d x=\frac{3}{4}\left(\nu-\frac{\mu}{3}\right) \int_{\Omega_{t}}\left(v_{i, x_{j}}+v_{j, x_{i}}\right)^{2} d x \\
& >0 \quad \text { for } \nu>\frac{1}{3} \mu .
\end{aligned}
$$

5. Global existence. We assume that

$$
f=0, \quad \theta_{1} \geq 0,
$$

and

$$
\|r\|_{0, \Omega_{t}}^{2}+|r|_{2,0, \Omega_{t}}^{2}+\|r\|_{0, \Omega_{t}}+\left|\theta_{1}\right|_{4,1, \Omega_{t}}^{2}+\left\|\theta_{1}\right\|_{1, \Omega_{t}} \leq \eta_{1} e^{-\eta_{2} t}
$$

where $\eta_{1}>0$ is sufficiently small and $\eta_{2}>1$.

Let $\varphi(t)$ and $\Phi(t)$ be defined by (4.5). We introduce the spaces

$$
\begin{aligned}
\mathfrak{N}(t) & =\left\{\left(v, \vartheta_{0}, \varrho_{\sigma}, \bar{\varrho}_{\Omega_{t}}\right): \varphi(t)<\infty\right\}, \\
\mathfrak{M}(t) & =\left\{\left(v, \vartheta_{0}, \vartheta, \varrho_{\sigma}, \bar{\varrho}_{\Omega_{t}}\right): \varphi(t)+\int_{0}^{t} \Phi\left(t^{\prime}\right) d t^{\prime}<\infty\right\} .
\end{aligned}
$$

Notice that $\left(v, \vartheta_{0}, \varrho_{\sigma}, \bar{\varrho}_{\Omega_{t}}\right) \in \mathfrak{N}(t)$ iff $\bar{\varphi}(t)<\infty$ and $\left(v, \vartheta_{0}, \vartheta, \varrho_{\sigma}, \bar{\varrho}_{\Omega_{t}}\right) \in$ $\mathfrak{M}(t)$ iff $\bar{\varphi}(t)+\int_{0}^{t} \Phi\left(t^{\prime}\right) d t^{\prime}<\infty$. Moreover, $c^{\prime} \varphi(t) \leq \bar{\varphi}(t) \leq c^{\prime \prime} \varphi(t)$, where $c^{\prime}, c^{\prime \prime}>0$ are constants.

LEMMA 5.1. Let the assumptions of Theorem 3.1 be satisfied. Let the initial data $v_{0}, \varrho_{0}, \theta_{0}, S$ of problem $(1.1)$ be such that $\left(v, \vartheta_{0}, \varrho_{\sigma}, \bar{\varrho}_{\Omega_{t}}\right) \in \mathfrak{N}(0)$ and $S \in W_{2}^{4-1 / 2}$. Let

$$
\int_{\Omega} \varrho_{0} v_{0} d \xi=0, \quad \int_{\Omega} \varrho_{0} \xi d \xi=0 .
$$

Moreover, assume

$$
\bar{\varphi}(0) \leq \varepsilon_{1},
$$

where $\varepsilon_{1}$ is sufficiently small. Then the local solution $(v, \theta, \varrho)$ of problem (1.1) is such that $\left(v, \vartheta_{0}, \vartheta, \varrho_{\sigma}, \bar{\varrho}_{\Omega_{t}}\right) \in \mathfrak{M}(t)$ for $t \leq T$, where $T$ is the time of local existence and

$$
\varphi(t)+\int_{0}^{t} \Phi\left(t^{\prime}\right) d t^{\prime} \leq c \varepsilon_{1} .
$$


Pro of. Take $\left(v, \vartheta_{0}, \varrho_{\sigma}, \bar{\varrho}_{\Omega_{t}}\right) \in \mathfrak{N}(0), S \in W_{2}^{4-1 / 2}$. Then $\left(v_{0}, \vartheta_{00}, \varrho_{\sigma 0}\right) \in$ $W_{2}^{3}(\Omega)\left(\varrho_{\sigma 0}=\varrho_{0}-\varrho_{\mathrm{e}}, \vartheta_{00}=\theta_{0}-\theta_{\mathrm{e}}\right)$ and by Theorem 3.1, Remark 3.2 and (5.2) there exists a solution of problem (1.1) such that

$$
\begin{aligned}
& u \in W_{2}^{4,2}\left(\Omega^{T}\right), \quad \vartheta_{0} \in W_{2}^{4,2}\left(\Omega^{T}\right), \\
& \eta_{\sigma} \in W_{2}^{3,3 / 2}\left(\Omega^{T}\right) \cap C^{0}\left(0, T ; \Gamma_{0}^{3,3 / 2}(\Omega)\right)
\end{aligned}
$$

and

$$
\begin{aligned}
\|u\|_{4, \Omega^{T}}^{2}+\left\|\eta_{\sigma}\right\|_{3, \Omega^{T}}^{2}+\mid \eta_{\sigma} \mathbf{I}_{3,0, \infty, \Omega^{T}}^{2}+\left\|\gamma_{0}\right\|_{4, \Omega^{T}}^{2} \\
\leq c\left(\left\|v_{0}\right\|_{3, \Omega}^{2}+\left\|\varrho_{\sigma 0}\right\|_{3, \Omega}^{2}+\left\|\vartheta_{00}\right\|_{3, \Omega}^{2}\right) \leq c \bar{\varphi}(0) \leq c \varepsilon_{1},
\end{aligned}
$$

where $u=v(x(\xi, t), t), \eta_{\sigma}=\varrho_{\sigma}(x(\xi, t), t), \gamma_{0}=\vartheta_{0}(x(\xi, t), t)$.

Using estimate (5.6) for the local solution and the imbeddings (see Lemmas 2.2 and 2.1)

$\sup _{t}\left(\|u\|_{3, \Omega}^{2}+\left\|u_{t}\right\|_{1, \Omega}^{2}\right) \leq c\left(\|u\|_{4, \Omega^{T}}^{2}+\|u(0)\|_{3, \Omega}^{2}+|u(0)|_{1,0, \Omega}^{2}\right) \leq c \bar{\varphi}(0) \leq c \varepsilon_{1}$ and

$$
\int_{0}^{t}\left|u_{\xi}\right|_{\infty, \Omega} d t^{\prime} \leq c T^{1 / 2}\|u\|_{4, \Omega^{T}} \leq c T^{1 / 2} \bar{\varphi}(0)
$$

we have the following estimate for the solution $\eta_{\sigma}$ of $(3.5)_{2}$ (see [22], Lemma 6.1):

$$
\begin{aligned}
N_{1} \equiv & \sup _{t}\left(\left\|\eta_{\sigma t t}\right\|_{0, \Omega}^{2}+\left\|\eta_{\sigma t}\right\|_{2, \Omega}^{2}+\left\|\eta_{\sigma}\right\|_{3, \Omega}^{2}\right) \\
& +\left\|\eta_{\sigma t t}\right\|_{L_{2}\left(0, T ; W_{2}^{1}(\Omega)\right)}^{2}+\left\|\eta_{\sigma t}\right\|_{L_{2}\left(0, T ; W_{2}^{3}(\Omega)\right)}^{2} \\
\leq & \varphi_{1}(T, \varphi(0)) \leq c \varepsilon_{1},
\end{aligned}
$$

where $\varphi_{1}$ is an increasing continuous function of its arguments.

Repeating the proof of Lemma 3.10 of [19] we get

$$
\begin{aligned}
& \frac{1}{2} \frac{d}{d t} \int_{\Omega_{t}}\left(\varrho v_{x x t}^{2}+\frac{p_{\sigma \varrho}}{\varrho} \varrho_{\sigma x x t}^{2}+\frac{\varrho c_{v}}{\theta} \vartheta_{0 x x t}^{2}\right) d x \\
& \quad+C\left(\left\|v_{x x t}\right\|_{1, \Omega_{t}}^{2}+\left\|\varrho_{\sigma x x t}\right\|_{0, \Omega_{t}}^{2}+\left\|\vartheta_{0 x x t}\right\|_{1, \Omega_{t}}^{2}\right) \\
& \leq\left(\varepsilon_{1}^{\prime}+c N\right)\left(\left\|v_{x t t t}\right\|_{0, \Omega_{t}}^{2}+\left\|v_{x x x t}\right\|_{0, \Omega_{t}}^{2}+\left\|v_{x x t t}\right\|_{0, \Omega_{t}}^{2}+\left\|\vartheta_{0 x t t t}\right\|_{0, \Omega_{t}}^{2}\right. \\
& \left.\quad+\left\|\vartheta_{0 x x t t}\right\|_{0, \Omega_{t}}^{2}+\left\|\vartheta_{0 x x x t}\right\|_{0, \Omega_{t}}^{2}\right)+c M(1+N)^{2}+c F(t),
\end{aligned}
$$

where $C, c>0$ are constants, $N=N_{1}+N_{2}, N_{2}=\sup _{t}\left(\|u\|_{3, \Omega}^{2}+\left\|u_{t}\right\|_{1, \Omega}^{2}+\right.$ $\left.\left\|\gamma_{0}\right\|_{3, \Omega}^{2}+\left\|\gamma_{0 t}\right\|_{1, \Omega}^{2}\right)$ and $M$ is such that $\int_{0}^{T} M d t^{\prime} \leq c \bar{\varphi}(0)$ holds in view of the estimates for the local solution. 
Similarly, using Lemma 3.11 of [19] yields

$$
\begin{aligned}
\frac{1}{2} \frac{d}{d t} \int_{\Omega_{t}} & \left(\varrho v_{x x t}^{2}+\frac{p_{\sigma \varrho}}{\varrho} \varrho_{\sigma x x t}^{2}+\frac{\varrho c_{v}}{\theta} \vartheta_{0 x x t}^{2}\right) d x \\
& +C\left(\left\|v_{x t t}\right\|_{1, \Omega_{t}}^{2}+\left\|\varrho_{\sigma x t t}\right\|_{0, \Omega_{t}}^{2}+\left\|\vartheta_{0 x t t}\right\|_{1, \Omega_{t}}^{2}\right) \\
\leq & \left(\varepsilon_{2}^{\prime}+c N\right)\left(\left\|v_{x t t t}\right\|_{0, \Omega_{t}}^{2}+\left\|v_{x x t t}\right\|_{0, \Omega_{t}}^{2}+\left\|v_{x x x t}\right\|_{0, \Omega_{t}}^{2}\right. \\
& \left.+\left\|\vartheta_{0 x t t t}\right\|_{0, \Omega_{t}}^{2}+\left\|\vartheta_{0 x x t t}\right\|_{0, \Omega_{t}}^{2}+\left\|\vartheta_{0 x x x t}\right\|_{0, \Omega_{t}}^{2}\right)+c(1+N)^{2} \\
& \times\left(\left\|v_{x x t}\right\|_{1, \Omega_{t}}^{2}+\left\|\vartheta_{0 x x t}\right\|_{1, \Omega_{t}}^{2}\right)+c M(1+N)^{2}+c F(t) .
\end{aligned}
$$

Next, Lemma 3.12 of [19] implies

$$
\begin{aligned}
\frac{1}{2} \frac{d}{d t} \int_{\Omega_{t}} & \left(\varrho v_{t t t}^{2}+\frac{p_{\sigma \varrho}}{\varrho} \varrho_{\sigma t t t}^{2}+\frac{\varrho c_{v}}{\theta} \vartheta_{0 t t t}^{2}\right) d x \\
& +C\left(\left\|v_{t t t}\right\|_{1, \Omega_{t}}^{2}+\left\|\varrho_{\sigma t t t}\right\|_{0, \Omega_{t}}^{2}+\left\|\vartheta_{0 t t t}\right\|_{1, \Omega_{t}}^{2}\right) \\
\leq & \left(\varepsilon_{3}^{\prime}+N+M\right)\left(\left\|v_{t t t}\right\|_{0, \Omega_{t}}^{2}+\left\|\vartheta_{0 t t t}\right\|_{0, \Omega_{t}}^{2}\right) \\
& +c N\left(\left\|v_{x t t}\right\|_{1, \Omega_{t}}^{2}+\left\|v_{x t t t}\right\|_{0, \Omega_{t}}^{2}+\left\|v_{x x t}\right\|_{1, \Omega_{t}}^{2}+\left\|\vartheta_{0 x t t}\right\|_{1, \Omega_{t}}^{2}\right. \\
& \left.+\left\|\vartheta_{0 x t t t}\right\|_{0, \Omega_{t}}^{2}+\left\|\vartheta_{0 x x t}\right\|_{1, \Omega_{t}}^{2}+\left\|\varrho_{\sigma t t t}\right\|_{0, \Omega_{t}}^{2}\right)+c\left\|v_{x t t}\right\|_{1, \Omega_{t}}^{2} \\
& +c\left\|\vartheta_{0 x t t}\right\|_{1, \Omega_{t}}^{2}+c M(1+N)^{2}+c F(t),
\end{aligned}
$$

where in virtue of the continuity equation $(4.4)_{2}$ we have

$$
\left\|\varrho_{\sigma t t t}\right\|_{0, \Omega_{t}}^{2} \leq c(1+N)\left\|v_{x t t}\right\|_{0, \Omega_{t}}^{2}+c M(1+N)^{2} .
$$

Finally, to estimate $\left\|\bar{\varrho}_{\Omega_{t}}\right\|_{0, \Omega_{t}}^{2}+\|\vartheta\|_{0, \Omega_{t}}^{2}$ rewrite the equation

$$
p\left(\varrho_{\Omega_{t}}, \theta_{\Omega_{t}}\right)-p\left(\varrho_{\mathrm{e}}, \theta_{\mathrm{e}}\right)=0
$$

using the Taylor formula as

$$
p_{\varrho}\left(\varrho_{\Omega_{t}}-\varrho_{\mathrm{e}}\right)+p_{\theta}\left(\theta_{\Omega_{t}}-\theta_{\mathrm{e}}\right)=0
$$

Hence

$$
\begin{aligned}
& \left\|\bar{\varrho}_{\Omega_{t}}\right\|_{0, \Omega_{t}}^{2}+\|\vartheta\|_{0, \Omega_{t}}^{2} \\
\leq & \left\|\varrho_{\sigma}\right\|_{0, \Omega_{t}}^{2}+\left\|\varrho_{\mathrm{e}}-\varrho_{\Omega_{t}}\right\|_{0, \Omega_{t}}^{2}+\left\|\vartheta_{0}\right\|_{0, \Omega_{t}}^{2}+\left\|\theta_{\mathrm{e}}-\theta_{\Omega_{t}}\right\|_{0, \Omega_{t}}^{2} \\
\leq & c\left(\left\|\varrho_{\sigma}\right\|_{0, \Omega_{t}}^{2}+\left\|\vartheta_{0}\right\|_{0, \Omega_{t}}^{2}+\left\|\theta_{\mathrm{e}}-\theta_{\Omega_{t}}\right\|_{0, \Omega_{t}}^{2}\right) \\
\leq & c\left(\left\|\varrho_{\sigma}\right\|_{0, \Omega_{t}}^{2}+\left\|\vartheta_{0}\right\|_{0, \Omega_{t}}^{2}+\left\|\frac{1}{\left|\Omega_{t}\right|} \int_{\Omega_{t}} \vartheta_{0} d x\right\|_{0, \Omega_{t}}^{2}\right) \leq c \bar{\varphi}(0) \leq c \varepsilon_{1}
\end{aligned}
$$

where to estimate $\left\|\varrho_{\sigma}\right\|_{0, \Omega_{t}}^{2}$ and $\left\|\vartheta_{0}\right\|_{0, \Omega_{t}}^{2}$ we have used (5.6). 
From (5.8)-(5.12) and (5.1)-(5.2), for sufficiently small $\varepsilon_{1}^{\prime}, \varepsilon_{2}^{\prime}, \varepsilon_{3}^{\prime}, N$, $\int_{0}^{T} M d t^{\prime}$ and $\eta_{1}$ from (5.2), we deduce that $\left(v(t), \varrho_{\sigma}(t), \bar{\varrho}_{\Omega_{t}}(t), \vartheta_{0}(t), \vartheta(t)\right) \in$ $\mathfrak{M}(t)$ for $t \leq T$ and (5.4) is satisfied. Of course to prove the last statement the standard technique of mollifiers or differences should be used. This concludes the proof.

LEMMA 5.2. Assume that there exists a local solution to problem (1.1) which belongs to $\mathfrak{M}(t)$ for $t \leq T$, i.e. let the assumptions of Lemma 5.1 be satisfied. Let the assumptions of Lemma 2.3 be satisfied. Then there exist $\delta_{1}, \delta_{2} \in(0,1)$ sufficiently small such that

$$
\begin{gathered}
\left\|p_{\sigma}\right\|_{0, \Omega_{t}}^{2} \leq \delta_{1}, \\
\left\|\vartheta_{0}\right\|_{0, \Omega_{t}}^{2}+\left\|\varrho_{\sigma}\right\|_{0, \Omega_{t}}^{2} \leq \delta_{2},
\end{gathered}
$$

where $\delta_{1}=c \varepsilon_{1} \delta^{\prime}+c\left(\delta^{\prime}\right) \widetilde{\delta}, \delta_{2}=c \varepsilon_{1} \delta^{\prime}+c\left(\delta^{\prime}\right)\left(\delta_{0}+\widetilde{\delta}\right), \delta^{\prime} \in(0,1)$ is as small as needed, $c\left(\delta^{\prime}\right)$ is a decreasing function of $\delta^{\prime}$, and $\delta_{0}$ and $\widetilde{\delta}$ are taken from Lemma 2.3.

Proof. Estimate (5.13) can be proved in exactly the same way as estimate $(6.13)$ in [20]. In order to prove $(5.14)$ we use the relation

$$
|\Omega|-\left|\Omega_{\mathrm{e}}\right|=\frac{1}{\varrho_{\mathrm{e}}} \int_{\Omega}\left(\varrho_{\mathrm{e}}-\varrho_{0}\right) d \xi .
$$

Hence, by assumption (2.8) of Lemma 2.3 we have

$$
\left\|\Omega|-| \Omega_{\mathrm{e}}\right\| \leq c \delta_{0}
$$

Using (5.15) and Remark 2.4 we obtain

$$
|| \Omega_{t}|-| \Omega_{\mathrm{e}} \| \leq c \delta
$$

where $\delta=\delta\left(\delta_{0}\right) \rightarrow 0$ as $\delta_{0} \rightarrow 0$.

If $\varrho>\varrho_{\mathrm{e}}$ then $\left(1 / \varrho_{\mathrm{e}}\right) \int_{\Omega_{t}}\left|\varrho-\varrho_{\mathrm{e}}\right| d x=\left|\Omega_{\mathrm{e}}\right|-\left|\Omega_{t}\right|$.

If $\varrho<\varrho_{\mathrm{e}}$ then $\left(1 / \varrho_{\mathrm{e}}\right) \int_{\Omega_{t}}\left|\varrho-\varrho_{\mathrm{e}}\right| d x=\left|\Omega_{t}\right|-\left|\Omega_{\mathrm{e}}\right|$.

Therefore, from (5.16) it follows that

Hence

$$
\int_{\Omega_{t}}\left(\varrho-\varrho_{\mathrm{e}}\right)^{2} d x \leq c \int_{\Omega_{t}}\left|\varrho-\varrho_{\mathrm{e}}\right| d x \leq c \delta .
$$

$$
\left\|\varrho_{\sigma}\right\|_{0, \Omega_{t}} \leq \delta_{2}^{\prime}
$$

Using the Taylor formula we have

$$
p_{\sigma}=p_{1} \varrho_{\sigma}+p_{2} \vartheta_{0}
$$

where $p_{1}=p_{1}(\varrho, \theta)$ and $p_{2}=p_{2}\left(\varrho_{\mathrm{e}}, \theta\right)$ (see (3.4) of [19]). Now estimates (5.13), (5.18) and formula (5.19) yield

$$
\left\|\vartheta_{0}\right\|_{0, \Omega_{t}} \leq \delta_{2}^{\prime \prime} \text {. }
$$

By (5.19) and (5.20) we get (5.14). 
Lemma 5.3. Assume that there exists a local solution of (1.1) in $\mathfrak{M}(t)$ for $0 \leq t \leq T$. Let the assumptions of Lemma 2.3 be satisfied. Assume that the initial data are in $\mathfrak{N}(0)$ and

$$
\bar{\varphi}(0) \leq \gamma, \quad \gamma \in(0,1 / 2],
$$

where $\gamma$ is sufficiently small. Then the solution at $t \in[0, T]$ belongs to $\mathfrak{N}(t)$ and

$$
\bar{\varphi}(t) \leq \gamma
$$

P r o o f. Assumption (5.21) and Lemma 5.1 imply that the estimate (4.6) can be written as

$$
\frac{d \bar{\varphi}}{d t}+c_{0} \Phi \leq c_{1}^{\prime}\left(\varphi+\int_{0}^{t} \Phi\left(t^{\prime}\right) d t^{\prime}\right)\left(1+\varphi^{3}\right)(\varphi+\Phi)+c_{2} F+c_{3} \psi .
$$

Since $\Phi+\left\|\vartheta_{0}\right\|_{0, \Omega_{t}}^{2}+\left\|\varrho_{\sigma}\right\|_{0, \Omega_{t}}^{2} \geq \varphi$, using Lemma 5.2 we obtain

$$
\Phi+\delta_{2} \geq \varphi
$$

where $\delta_{2}$ is independent of $\gamma$.

Next, by Lemmas 5.2 and 2.3 and assumptions (5.1)-(5.2) we have

$$
F+\psi \leq \eta+\delta_{1}+c \widetilde{\delta}
$$

where $\eta=\eta_{1} e^{-\eta_{2} t}$ and $\delta_{1}, \widetilde{\delta}$ are sufficiently small. Using (5.24) in (5.23) gives

$$
\frac{d \bar{\varphi}}{d t}+c_{0} \Phi \leq c_{1}^{\prime}\left(\varphi+\int_{0}^{t} \Phi\left(t^{\prime}\right) d t^{\prime}\right)\left(1+\varphi^{3}\right)\left(2 \Phi+\delta_{2}\right)+c_{2} F+c_{3} \psi
$$

Assuming that the initial data are so small that

$$
2 c_{1}^{\prime}\left(\varphi+\int_{0}^{t} \Phi\left(t^{\prime}\right) d t^{\prime}\right)\left(1+\varphi^{3}\right) \Phi \leq \frac{c_{0} \Phi}{2},
$$

instead of (5.26) we get

$$
\frac{d \bar{\varphi}}{d t}+\frac{c_{0} \Phi}{2} \leq c_{1}^{\prime}\left(\varphi+\int_{0}^{t} \Phi\left(t^{\prime}\right) d t^{\prime}\right)\left(1+\varphi^{3}\right) \delta_{2}+c_{2} F+c_{3} \psi .
$$

Now using (5.24), (5.25), (5.21) and Lemma 5.1 in (5.27) yields

$$
\frac{d \bar{\varphi}}{d t}+\frac{c_{0} \Phi}{2} \leq c_{4}\left(\gamma \delta_{2}+\frac{\delta_{2}}{2}+\eta+\delta_{1}+c \widetilde{\delta}\right) .
$$

By (5.21), $\bar{\varphi}(0) \leq \gamma, \gamma \in(0,1 / 2]$. Assume that $t_{*}=\inf \{t \in[0, T]: \bar{\varphi}(t)>$ $\gamma$ \}. Consider (5.28) in the interval $\left[0, t_{*}\right]$. From the definition of $t_{*}$ we have 
$\bar{\varphi}\left(t_{*}\right)=\gamma$. Therefore $(5.28)$ implies

$$
\bar{\varphi}_{t}\left(t_{*}\right) \leq-\frac{\gamma}{2}+c_{4}\left(\gamma \delta_{2}+\frac{\delta_{2}}{2}+\eta+\delta_{1}+c \widetilde{\delta}\right) .
$$

Assume that $\delta_{2}, \eta, \delta_{1}$ and $\widetilde{\delta}$ are so small that

$$
c_{4}\left(\gamma \delta_{2}+\frac{\delta_{2}}{2}+\eta+\delta_{1}+c \widetilde{\delta}\right)<\frac{\gamma}{4} .
$$

Hence (5.29) yields $\bar{\varphi}_{t}\left(t_{*}\right)<0$, a contradiction. Therefore (5.22) holds.

Lemma 5.3 suggests that the solution can be continued to the interval $[T, 2 T]$, but to do this we need the following facts:

(5.30) (a) The existence of the transformation $x=x(\xi, t)$ and its inverse for $t \in[T, 2 T]$.

(b) The validity of the Korn inequality with the same constant for the whole interval $[0,2 T]$.

(c) The variations of the shape of $\Omega_{t}$ for $t \in[0,2 T]$ are so small that the constants in Lemma 2.1 (imbedding (2.1)) can be chosen independently of $t$.

Generally, to prove the global existence we need these facts for all $t$. Theorem 2.7 of [18] implies that the volume of $\Omega_{t}$ does not change much but we have not shown yet any restriction on the variations of its shape.

It is sufficient to show (c), because (a) and (b) follow.

LEMMA 5.4. Assume that there exists a local solution of (1.1) in $\mathfrak{M}(t)$ for $0 \leq t \leq T$ with initial data in $\mathfrak{N}(0)$ sufficiently small (see (5.3)). Then there exist constants $\mu_{1}>0$ and $\mu_{2}>0\left(\mu_{2}\right.$ is sufficiently small) such that

$$
\bar{\varphi}(t) \leq c e^{-\mu_{1} t}\left(\bar{\varphi}(0)+\mu_{2}\right), \quad t \leq T,
$$

where $c>0$ is a constant and $T$ is the time of local existence. Moreover, if we assume (5.2) with $\eta_{1}=0$, then (5.31) holds with $\mu_{2}=0$.

P r o of. Inequalities (3.20) and (3.28) of [19] (see the proof of Lemma 3.1 and the assertion of Lemma 3.2 of [19]) imply

$$
\begin{aligned}
\frac{d}{d t} \int_{\Omega_{t}}\left[\varrho \left(v^{2}\right.\right. & \left.\left.+v_{t}^{2}\right)+\frac{1}{\varrho}\left(p_{1} \varrho_{\sigma}^{2}+p_{\varrho} \varrho_{\sigma t}^{2}\right)+\frac{\varrho c_{v}}{\theta}\left(\frac{p_{2}}{p_{\theta}} \vartheta_{0}^{2}+\vartheta_{0 t}^{2}\right)\right] d x \\
& +c_{0}\left(\|v\|_{1, \Omega_{t}}^{2}+\left\|v_{t}\right\|_{1, \Omega_{t}}^{2}+\|\operatorname{div} v\|_{0, \Omega_{t}}^{2}+\left\|\operatorname{div} v_{t}\right\|_{0, \Omega_{t}}^{2}\right. \\
& \left.+\left\|\vartheta_{0 x}\right\|_{0, \Omega_{t}}^{2}+\left\|\vartheta_{0 t}\right\|_{1, \Omega_{t}}^{2}+\left\|\varrho_{\sigma t}\right\|_{0, \Omega_{t}}^{2}\right) \\
\leq & C_{2} \varphi^{2}(t)(1+\varphi(t))+C_{1} F(t) .
\end{aligned}
$$


Multiplying both sides of (5.32) by $e^{-\alpha t}$ (where $0<\alpha<1$ ) we obtain

$$
\begin{aligned}
& \frac{d}{d t}\left\{e ^ { - \alpha t } \int _ { \Omega _ { t } } \left[\varrho\left(v^{2}+v_{t}^{2}\right)+\frac{1}{\varrho}\left(p_{1} \varrho_{\sigma}^{2}+p_{\varrho} \varrho_{\sigma t}^{2}\right)\right.\right. \\
& \left.\left.\quad+\frac{\varrho c_{v}}{\theta}\left(\frac{p_{2}}{p_{\theta}} \vartheta_{0}^{2}+\vartheta_{0 t}^{2}\right)\right] d x\right\}+\alpha e^{-\alpha t} \int_{\Omega_{t}}\left[\varrho\left(v^{2}+v_{t}^{2}\right)\right. \\
& \left.\quad+\frac{1}{\varrho}\left(p_{1} \varrho_{\sigma}^{2}+p_{\varrho} \varrho_{\sigma t}^{2}\right)+\frac{\varrho c_{v}}{\theta}\left(\frac{p_{2}}{p_{\theta}} \vartheta_{0}^{2}+\vartheta_{0 t}^{2}\right)\right] d x \\
& \quad+c_{0} e^{-\alpha t}\left(\|v\|_{1, \Omega_{t}}^{2}+\left\|v_{t}\right\|_{1, \Omega_{t}}^{2}+\|\operatorname{div} v\|_{0, \Omega_{t}}^{2}+\left\|\operatorname{div} v_{t}\right\|_{0, \Omega_{t}}^{2}\right. \\
& \left.\quad+\left\|\vartheta_{0 x}\right\|_{0, \Omega_{t}}^{2}+\left\|\vartheta_{0 t}\right\|_{1, \Omega_{t}}^{2}+\left\|\varrho_{\sigma t}\right\|_{0, \Omega_{t}}^{2}\right) \\
& \leq C_{2} e^{-\alpha t} \varphi^{2}(t)(1+\varphi(t))+C_{1} e^{-\alpha t} F(t) .
\end{aligned}
$$

Next multiplying inequality (4.6) by $e^{-\alpha t}$ we have

$$
\begin{aligned}
\frac{d \bar{\varphi}_{1}}{d t}+ & \alpha \varphi_{1}+c_{0} \Phi_{1} \\
\leq & c_{1} P(\varphi)\left(\varphi+\int_{0}^{t}\|v\|_{4, \Omega_{t^{\prime}}}^{2} d t^{\prime}\right)\left(1+\varphi^{3}\right)\left(\varphi_{1}+\Phi_{1}\right) \\
& +c_{2} F_{1}+c_{3} \psi_{1},
\end{aligned}
$$

where $\varphi_{1}=\varphi e^{-\alpha t}, \bar{\varphi}_{1}=\bar{\varphi} e^{-\alpha t}, \Phi_{1}=\Phi e^{-\alpha t}, F_{1}=F e^{-\alpha t}$, and $\psi_{1}=\psi e^{-\alpha t}$.

From the assumption that the initial data are sufficiently small we deduce that $\varphi+\int_{0}^{t}\|v\|_{4, \Omega_{t^{\prime}}}^{2} d t^{\prime}$ is also small (see Lemma 5.1). Therefore, for sufficiently small data from (5.34) we get

$$
\frac{d \bar{\varphi}_{1}}{d t}+c_{4}\left(\varphi_{1}+\Phi_{1}\right) \leq c_{5} F_{1}+c_{6}\left(\left\|p_{\sigma}\right\|_{0, \Omega_{t}}^{2}+\|v\|_{0, \Omega_{t}}^{2}\right) e^{-\alpha t} .
$$

Now, applying the same argument as in the proof of Lemma 6.2 of [20] we obtain

$$
\left\|p_{\sigma}\right\|_{0, \Omega_{t}}^{2} \leq \varepsilon\left(\left\|p_{\sigma x}\right\|_{0, \Omega_{t}}^{2}+\left\|v_{x x}\right\|_{0, \Omega_{t}}^{2}\right)+c(\varepsilon)\left(\|v\|_{0, \Omega_{t}}^{2}+\left\|v_{t}\right\|_{0, \Omega_{t}}^{2}\right) .
$$

Moreover,

$$
\left\|p_{\sigma x}\right\|_{0, \Omega_{t}}^{2} \leq c\left(\left\|\varrho_{\sigma x}\right\|_{0, \Omega_{t}}^{2}+\left\|\vartheta_{0 x}\right\|_{0, \Omega_{t}}^{2}\right) .
$$

Using (5.36) and (5.37) in (5.35) we have

$$
\frac{d \bar{\varphi}_{1}}{d t}+c_{4}\left(\varphi_{1}+\Phi_{1}\right) \leq c_{5} F_{1}+c_{7}\left(\|v\|_{0, \Omega_{t}}^{2}+\left\|v_{t}\right\|_{0, \Omega_{t}}^{2}\right) e^{-\alpha t} .
$$

Multiplying (5.33) by a sufficiently large constant $c_{8}$, adding the result to 
(5.38) and using the fact that $\bar{\varphi}(0)$ is sufficiently small we obtain

$$
\frac{d \widetilde{\bar{\varphi}}}{d t}+c_{9}(\widetilde{\varphi}+\widetilde{\Phi}) \leq c_{10} F_{1},
$$

where

$$
\begin{aligned}
\widetilde{\varphi}= & \varphi_{1}+c_{8} e^{-\alpha t} \int_{\Omega_{t}}\left[\varrho\left(v^{2}+v_{t}^{2}\right)+\frac{1}{\varrho}\left(p_{1} \varrho_{\sigma}^{2}+p_{\sigma} \varrho_{\sigma t}^{2}\right)\right. \\
& \left.+\frac{\varrho c_{v}}{\theta}\left(\frac{p_{2}}{p_{\theta}} \vartheta_{0}^{2}+\vartheta_{0 t}^{2}\right)\right] d x \\
\widetilde{\bar{\varphi}}= & \bar{\varphi}_{1}+c_{8} e^{-\alpha t} \int_{\Omega_{t}}\left[\varrho\left(v^{2}+v_{t}^{2}\right)+\frac{1}{\varrho}\left(p_{1} \varrho_{\sigma}^{2}+p_{\sigma} \varrho_{\sigma t}^{2}\right)\right. \\
& \left.+\frac{\varrho c_{v}}{\theta}\left(\frac{p_{2}}{p_{\theta}} \vartheta_{0}^{2}+\vartheta_{0 t}^{2}\right)\right] d x, \\
\widetilde{\Phi}= & \Phi_{1}+c_{8} e^{-\alpha t}\left(\|v\|_{1, \Omega_{t}}^{2}+\left\|v_{t}\right\|_{1, \Omega_{t}}^{2}+\|\operatorname{div} v\|_{0, \Omega_{t}}^{2}\right. \\
& \left.+\left\|\operatorname{div} v_{t}\right\|_{0, \Omega_{t}}^{2}+\left\|\vartheta_{0 x}\right\|_{0, \Omega_{t}}^{2}+\left\|\vartheta_{0 t}\right\|_{1, \Omega_{t}}^{2}+\left\|\varrho_{\sigma t}\right\|_{0, \Omega_{t}}^{2}\right) .
\end{aligned}
$$

There exist constants $c_{0}^{\prime}, c_{0}^{\prime \prime}>0$ such that

$$
c_{0}^{\prime} \bar{\varphi}_{1} \leq \widetilde{\bar{\varphi}} \leq c_{0}^{\prime \prime} \bar{\varphi}_{1} \quad \text { and } \quad c_{0}^{\prime} \Phi_{1} \leq \widetilde{\Phi} \leq c_{0}^{\prime \prime} \Phi_{1} .
$$

Hence by assumptions (5.1) and (5.2) inequality (5.39) implies

$$
\bar{\varphi}_{1} \leq c_{12} e^{-c_{10} t}\left(\bar{\varphi}(0)+c_{11}\right) \text {, }
$$

where $c_{11}>0$ is sufficiently small.

For $\alpha$ sufficiently small, from (5.40) we obtain (5.31).

Finally, we prove the main result of this paper.

THEOREM 5.5. Let $\nu>\frac{1}{3} \mu$. Let (5.1), (5.2) with $\eta_{1}=0$ and the assumptions of Theorem 3.1 with $r \in C_{B}^{2,1}\left(\mathbb{R}^{3} \times[0,+\infty)\right)$ and $\theta_{1} \in C_{B}^{2,1}\left(\mathbb{R}^{3} \times\right.$ $[0,+\infty))$ be satisfied. Furthermore, let $\left(v, \vartheta_{0}, \varrho_{\sigma}, \bar{\varrho}_{\Omega_{t}}\right) \in \mathfrak{N}(0)$ and

$$
\varphi(0) \leq \varepsilon_{1},
$$

where $\varepsilon_{1} \in(0,1)$ is sufficiently small. Let the following compatibility conditions be satisfied:

$$
\begin{array}{ll}
\left.D^{\alpha} \partial_{t}^{i}\left(\mathbb{T} \cdot \bar{n}+p_{0} \bar{n}\right)\right|_{t=0, S}=0, & |\alpha|+i \leq 2, \\
\left.D^{\alpha} \partial_{t}^{i}\left(\bar{n} \cdot \nabla \theta-\theta_{1}\right)\right|_{t=0, S}=0, & |\alpha|+i \leq 2 .
\end{array}
$$

Assume also that the internal energy per unit mass $\varepsilon=\varepsilon(\varrho, \theta)$ has the form (2.3) and conditions (2.4)-(2.5) hold. Moreover, assume that 


$$
\begin{gathered}
\int_{\Omega} \varrho_{0} \frac{v_{0}^{2}}{2} d \xi+\kappa \sup _{t} \int_{0}^{t} d t^{\prime} \int_{S_{t^{\prime}}} \theta_{1}\left(s, t^{\prime}\right) d s \\
+\int_{\Omega} \varrho_{0}\left(h\left(\varrho_{0}, \theta_{0}\right)-h_{*}\right) d \xi \leq \varepsilon_{2}, \\
\int_{\Omega}\left|\varrho_{0}-\varrho_{\mathrm{e}}\right| d \xi \leq \varepsilon_{2}, \\
\frac{(\beta-1)^{\beta-1}}{\beta^{\beta} p_{0}^{\beta-1}}\left(a_{0} \varrho_{\mathrm{e}}^{\beta}+p_{0}\right)^{\beta}-a_{0} \varrho_{\mathrm{e}}^{\beta} \leq \varepsilon_{2}, \\
a_{0}\left\{\int_{\Omega} \varrho_{0}^{\beta} d x-\frac{M^{\beta}\left(\beta p_{0}\right)^{\beta-1}}{\left[c \delta \beta \varrho_{0}+\left(d-h_{*} M\right)(\beta-1)\right]^{\beta-1}}\right\} \leq \varepsilon_{2},
\end{gathered}
$$

where $\varepsilon_{2}>0$ is a sufficiently small constant, $\beta=\alpha+1$, and $c>0$ and $\delta>0$ are the constants from Remark 2.6. Assume, finally, that

$$
\int_{\Omega} \varrho_{0} v_{0}(a+b \times \xi) d \xi=0, \quad \int_{\Omega} \varrho_{0} \xi d \xi=0, \quad \int_{\Omega} \varrho_{0} d \xi=M,
$$

where $a, b$ are arbitrary constant vectors. Then there exists a global solution of (1.1) such that $\left(v, \vartheta_{0}, \vartheta, \varrho_{\sigma}, \bar{\varrho}_{\Omega_{t}}\right) \in \mathfrak{M}(t)$ for $t \in \mathbb{R}_{+}^{1}$ and $S_{t} \in W_{2}^{4-1 / 2}$.

Proof. The theorem is proved step by step using the local existence in a fixed time interval. Under the assumption that

$$
\left(v, \vartheta_{0}, \varrho_{\sigma}, \bar{\varrho}_{\Omega_{t}}\right) \in \mathfrak{N}(0),
$$

Theorem 3.1 and Remark 3.2 yield the local existence of solutions of (1.1) such that

$$
\begin{aligned}
& u \in W_{2}^{4,2}\left(\Omega^{T}\right), \quad \vartheta_{0} \in W_{2}^{4,2}\left(\Omega^{T}\right), \\
& \eta_{\sigma} \in W_{2}^{3,3 / 2}\left(\Omega^{T}\right) \cap C^{0}\left(0, T ; \Gamma_{0}^{3,3 / 2}(\Omega)\right),
\end{aligned}
$$

where $T$ is the time of the existence. By (5.48) and (5.49), Lemma 5.1 implies that the local solution belongs to $\mathfrak{M}(t)$ for $t \leq T$. For small $\varepsilon_{1}$ the existence time $T$ is correspondingly large, so we can assume it is a fixed positive number.

To prove the last result we needed the Korn inequalities (see [20]) and Lemma 2.1 (imbedding (2.2)). The constants in those theorems depend on $\Omega_{t}$ and the shape of $S_{t}$, so generally they are functions of $t$. In view of (5.41), Lemma 5.1 gives

$$
\varphi(t)+\int_{0}^{t} \Phi\left(t^{\prime}\right) d t^{\prime} \leq c \varepsilon_{1}
$$


Hence we obtain

$$
\left|\int_{0}^{t} v d t^{\prime}\right| \leq c \varepsilon_{1}, \quad t \in[0, T] .
$$

Therefore from the relation

$$
x=\xi+\int_{0}^{t} u\left(\xi, t^{\prime}\right) d t^{\prime}, \quad \xi \in S, t \in T,
$$

it follows that for sufficiently small $\varepsilon_{1}$ and fixed $T$, the shape of $\Omega_{t}, t \leq T$, does not change too much, so the constants from the imbedding Lemma 2.1 can be chosen independent of time.

Since $\bar{\varphi}(t) \leq c^{\prime \prime} \varphi(t),(5.41)$, Lemma 5.3 and Remarks 2.5-2.6 imply

$$
\bar{\varphi}(T) \leq c^{\prime \prime} \varepsilon_{1},
$$

for sufficiently small $\varepsilon_{1}$ and $\varepsilon_{2}$.

Now we wish to extend the solution to the interval $[T, 2 T]$. Using (5.52) we can prove the existence of local solutions in $\mathfrak{M}(t)$ for $T \leq t \leq 2 T$. To prove

$$
\bar{\varphi}(2 T) \leq c^{\prime \prime} \varepsilon_{1}
$$

we need inequality (4.6), where the constants depend on the constants from the imbedding theorems and the Korn inequalities for $t \in[T, 2 T]$. Therefore, we have to show that the shape of $S_{t}, t \leq 2 T$, does not change more that for $t \leq T$. For this we need the following (see (5.30)). Assume that there exists a local solution in $[0, k T]$. Then in view of Lemma 5.4 , for $t \in[0, k T]$ we have

$$
\begin{aligned}
\left|\int_{0}^{t} v d x\right|+\left|\int_{0}^{t} v_{x} d x\right| \\
\quad \leq c_{2} \int_{0}^{t}\|v\|_{3, \Omega_{t^{\prime}}} d t^{\prime} \leq c_{2} \sum_{i=0}^{k-1} \int_{i T}^{(i+1) T}\|v\|_{3, \Omega_{t^{\prime}}} d t^{\prime} \\
\quad \leq c_{2} T^{1 / 2} \sum_{i=0}^{k-1}\left(\int_{i T}^{(i+1) T}\|v\|_{3, \Omega_{t^{\prime}}}^{2} d t^{\prime}\right)^{1 / 2} \leq c_{3} T^{1 / 2} \sum_{i=0}^{k-1}\left(\int_{i T}^{(i+1) T} \bar{\varphi}\left(t^{\prime}\right) d t^{\prime}\right)^{1 / 2} \\
\quad \leq c_{3} T^{1 / 2} \sum_{i=0}^{k-1}\left[\bar{\varphi}(i T) \int_{i T}^{(i+1) T} e^{-\mu_{1}(t-i T)} d t\right]^{1 / 2} \\
\quad \leq c_{3}\left[T\left(1-e^{-\mu_{1} T}\right) / \mu_{1}\right]^{1 / 2} \sum_{i=0}^{k-1}(\bar{\varphi}(i T))^{1 / 2}
\end{aligned}
$$




$$
\begin{aligned}
& \leq c_{3}\left[T\left(1-e^{-\mu_{1} T}\right) / \mu_{1}\right]^{1 / 2} \sum_{i=0}^{k-1}(\bar{\varphi}(i T))^{1 / 2} \\
& \leq c_{3}\left\{T\left[\left(1-e^{-\mu_{1} T}\right) / \mu_{1}\right] \bar{\varphi}(0)\left(1+e^{-\mu_{1} T}+e^{-2 \mu_{1} T}+\ldots\right)\right\}^{1 / 2} \\
& =c_{3}\left[T\left(1 / \mu_{1}\right) \bar{\varphi}(0)\left(1-e^{-\mu_{1} T}\right)\left(1-e^{-\mu_{1} T}\right)^{-1}\right]^{1 / 2} \\
& =c_{3}\left[T\left(1 / \mu_{1}\right) \bar{\varphi}(0)\right]^{1 / 2} \leq c_{4} T^{1 / 2} \varepsilon_{1}^{1 / 2} .
\end{aligned}
$$

Taking $k=2$ and $\varepsilon_{1}$ sufficiently small we see that $\left|\int_{0}^{t} v\left(x, t^{\prime}\right) d t^{\prime}\right|$ is small for any $t \in[T, 2 T]$, so (5.51) implies that the shape of $S_{t}$ changes no more than in $[0, T]$ and then the differential inequality (4.6) can be shown for this interval with the same constants, too. Hence in view of Lemma 5.1 the solution of (1.1) belongs to $\mathfrak{M}(t)$ for $t \in[T, 2 T]$. Next Lemmas 5.1-5.3 and Remarks 2.5-2.6 imply (5.53).

Repeating the above considerations for the intervals $[k T,(k+1) T], k \geq 2$, we prove the existence for all $t \in \mathbb{R}_{+}^{1}$. This concludes the proof of the theorem.

Remark 5.6. Lemma 5.4 implies that $\varphi(t) \rightarrow 0$ as $t \rightarrow \infty$. Hence the considered motion converges to the constant state.

\section{References}

[1] J. T. Beale, The initial value problem for the Navier-Stokes equations with a free boundary, Comm. Pure Appl. Math. 31 (1980), 359-392.

[2] - Large time regularity of viscous surface waves, Arch. Rational Mech. Anal. 84 (1984), 307-352.

[3] O. V. Besov, V. P. Il'in and S. M. Nikol'skiǔ, Integral Representations of Functions and Imbedding Theorems, Nauka, Moscow, 1975 (in Russian); English transl.: Scripta Series in Mathematics, Winston and Halsted Press, 1979.

[4] L. Landau and E. Lifschitz, Mechanics of Continuum Media, Nauka, Moscow, 1984 (in Russian); English transl.: Pergamon Press, Oxford, 1959; new edition: Hydrodynamics, Nauka, Moscow, 1986 (in Russian).

[5] A. Matsumura and T. Nishida, The initial value problem for the equations of motion of viscous and heat-conductive gases, J. Math. Kyoto Univ. 20 (1980), 67104.

[6] - - - The initial value problem for the equations of motion of compressible viscous and heat-conductive fluids, Proc. Japan Acad. Ser. A 55 (1979), 337-342.

[7] -, - , The initial boundary value problem for the equations of motion of compressible viscous and heat-conductive fluid, preprint of Univ. of Wisconsin, MRC Technical Summary Report no. 2237 (1981).

[8] -, -, Initial boundary value problems for the equations of motion of general fluids, in: Computing Methods in Applied Sciences and Engineering, R. Glowinski and J. L. Lions (eds.), North-Holland, Amsterdam, 1982, 389-406.

[9] - - - Initial boundary value problems for the equations of motion of compressible viscous and heat-conductive fluids, Comm. Math. Phys. 89 (1983), 445-464. 
[10] V. A. Solonnikov, A priori estimates for parabolic equations of second order, Trudy Mat. Inst. Steklov 70 (1964), 133-212 (in Russian).

[11] - On an unsteady flow of a finite mass of a liquid bounded by a free surface, Zap. Nauchn. Sem. LOMI 152 (1986), 137-157 (in Russian); English transl.: J. Soviet Math. 40 (1988), 672-686.

[12] - Solvability of the evolution problem for an isolated mass of a viscous incompressible capillary liquid, Zap. Nauchn. Sem. LOMI 140 (1984), 179-186 (in Russian); English transl.: J. Soviet Math. 32 (1986), 223-238.

[13] -, On an unsteady motion of an isolated volume of a viscous incompressible fluid, Izv. Akad. Nauk SSSR Ser. Mat. 51 (1987), 1065-1087 (in Russian).

[14] V. A. Solonnikov and A. Tani, Evolution free boundary problem for equations of motion of viscous compressible barotropic liquid, preprint of Paderborn University.

[15] A. Valli and W. M. Zajaczkowski, Navier-Stokes equations for compressible fluids: global existence and qualitative properties of the solutions in the general case, Comm. Math. Phys. 103 (1986), 259-296.

[16] E. Zadrzyńska and W. M. Zajạczkowski, On local motion of a general compressible viscous heat conducting fluid bounded by a free surface, Ann. Polon. Math. 59 (1994), 133-170.

[17] —, - On global motion of a compressible viscous heat conducting fluid bounded by a free surface, Acta Appl. Math. 37 (1994), 221-231.

[18] - - - Conservation laws in free boundary problems for viscous compressible heat conducting fluids, Bull. Polish Acad. Sci. Tech. Sci. 42 (1994), 197-207.

[19] —, - On a differential inequality for equations of a viscous compressible heat conducting fluid bounded by a free surface, Ann. Polon. Math. 61 (1995), 141-188.

[20] W. M. Zajączkowski, On nonstationary motion of a compressible barotropic viscous fluid bounded by a free surface, Dissertationes Math. 324 (1993).

[21] - On local motion of a compressible barotropic viscous fluid bounded by a free surface, in: Partial Differential Equations, Banach Center Publ. 27, Part 2, Inst. Math., Polish Acad. Sci., 1992, 511-553.

[22] —, Existence of local solutions for free boundary problems for viscous compressible barotropic fluids, Ann. Polon. Math. 60 (1995), 255-287.

[23] - On nonstationary motion of a compressible barotropic viscous capillary fluid bounded by a free surface, SIAM J. Math. Anal. 25 (1994), 1-84.

Ewa Zadrzyńska

Wojciech Zajączkowski

Institute of Mathematics

Institute of Mathematics

and Operations Research

Military University of Technology

Polish Academy of Sciences

S. Kaliskiego 2

Śniadeckich 8

01-489 Warszawa, Poland

00-950 Warszawa, Poland 